\title{
BMJ Open Association between prelabour caesarean section and perinatal outcomes: analysis of demographic and health surveys from 26 low-income and middle-income countries
}

\author{
Newton Opiyo (D) , ${ }^{1}$ Saverio Bellizzi, ${ }^{2}$ Maria Regina Torloni (i) , ${ }^{3}$ Joao Paulo Souza, ${ }^{4}$ \\ Ana Pilar Betran ${ }^{1}$
}

To cite: Opiyo N, Bellizzi S, Torloni MR, et al. Association between prelabour caesarean section and perinatal outcomes: analysis of demographic and health surveys from 26 low-income and middleincome countries. BMJ Open 2022;12:e053049. doi:10.1136/ bmjopen-2021-053049

- Prepublication history and additional supplemental material for this paper are available online. To view these files, please visit the journal online (http://dx.doi.org/10.1136/ bmjopen-2021-053049).

Received 04 May 2021 Accepted 30 December 2021

Check for updates

(C) Author(s) (or their employer(s)) 2022. Re-use permitted under CC BY-NC. No commercial re-use. See rights and permissions. Published by BMJ.

For numbered affiliations see end of article.

Correspondence to

Dr Newton Opiyo;

opiyon@who.int

\section{ABSTRACT}

Objectives Caesarean section (CS) conducted before labour (prelabour CS (PLCS)), compared with vaginal birth, may pose additional maternal and perinatal risks. No multicountry analysis has examined PLCS in low-income and middle-income countries (LMICS). This study assessed rates, risk factors and associations of PLCS with perinatal outcomes in LMICs.

Design Population-based cross-sectional surveys. Setting Demographic and Health Surveys conducted between 2015 and 2018 in 26 LMICs (13 countries in Africa, 11 in Asia and 2 in the Americas).

Participants Women aged $15-49$ years with singleton term births.

Outcome measures Main outcomes were early neonatal mortality, neonatal mortality, early breastfeeding (within 1 hour of birth), skin-to-skin contact and duration of hospital stay.

Results 255227 women were included in the main analysis. Average rates of primary PLCS ranged from $1.3 \%$ in Zambia to $19.5 \%$ in Maldives. Median PLCS rate was $1.8 \%$ in the poorest versus $5.8 \%$ in the richest subgroups.

Higher maternal age, education, economic status and BMI, lower parity, urban residence, delivery in private hospitals, larger baby size, having health insurance, more antenatal care (ANC) visits, ANC by a doctor and ANC in private hospitals were associated with increased primary PLCS.

Across the 26 countries, primary PLCS, compared with vaginal delivery, was associated with increased neonatal mortality (adjusted odds ratio, aOR 1.2, $95 \% \mathrm{Cl} 1.0$ to 1.5), decreased early breastfeeding $(\mathrm{aOR} 0.4,95 \% \mathrm{Cl} 0.3$ to 0.5 ) and skin-to-skin contact (aOR $0.4,95 \% \mathrm{Cl} 0.3$ to $0.5)$ and longer hospital stay (aOR 6.6, $95 \% \mathrm{Cl} 5.9$ to 7.4$)$. No significant association was found for early neonatal mortality (aOR $1.2,95 \% \mathrm{Cl} 0.9$ to 1.5 ).

Conclusion Primary PLCS, compared with vaginal birth, is associated with adverse perinatal outcomes in singleton term pregnancies in LMICs. Caesarean births should be audited regularly to monitor trends, appropriateness and context-specific drivers of CS.

\section{Strengths and limitations of this study}

- This is the first study assessing national rates, risk factors and associations of prelabour caesarean section (CS) with perinatal outcomes. The analysis is based on a large sample ( $n=255227$ women) from 26 low-income and middle-income countries (LMICs).

- Detailed clinical data including the indication of prelabour CS were not reported in the Demographic and Health Surveys (DHSs). It was, therefore, not possible to distinguish between medically indicated and non-indicated prelabour CS in the analysis.

- The DHS is a cross-sectional survey, which precludes causal inference for the observed associations between prelabour CS and perinatal outcomes.

- We could not control for confounding bias by indication of prelabour CS. Given this limitation, caution should be exercised when interpreting the study results.

\section{INTRODUCTION}

Although the onset of labour is not a fully understood phenomenon, it is likely triggered by interactive, finely coordinated anatomical and neurohormonal processes. ${ }^{12}$ The result is that spontaneous labour onset in healthy, pregnant women at term usually occurs when both the mother and baby are at peak biophysical, neurohormonal and psychological readiness for birth and maternal-newborn transitions. $^{2}{ }^{3}$ These innate processes are intended to facilitate optimal postnatal adaptation of the mother and newborn.

Physiologic labour and birth benefits include optimising maternal-newborn transitions and postnatal adaptations, breastfeeding, mother-infant attachment and other short-term and long-term health outcomes. ${ }^{4}$ 
Many contemporary perinatal interventions conducted before labour onset disrupt inherent mechanisms of labour and parturition, reduce their benefits and expose mothers and newborns to additional risks. ${ }^{3}$

When medically justified, obstetric interventions (such as induction or augmentation of labour, caesarean section (CS)) can reduce maternal and perinatal mortality and morbidity. ${ }^{5}{ }^{6}$ However, for healthy women and babies who do not require them, these interventions can disrupt physiologic processes, deprive the dyad of benefits and increase maternal and neonatal morbidity, ${ }^{4}$ inducing epigenetic transgenerational changes. ${ }^{78}$

In the global context of escalating medicalisation, birth by CS is rising to unprecedented levels while not necessarily reaching women who really need the procedure. ${ }^{910}$ The rise in the use of CS extends to low-income and middle-income countries (LMICs), where overuse and underuse coexist, and where the rate of CS complications is higher. ${ }^{11} \mathrm{~A}$ caesarean section before spontaneous or induced labour (ie, prelabour CS) may be necessary due to clinical or obstetric complications (eg, maternal cardiopathy, pre-eclampsia or placental anomalies). However, where no clear medical indication exists, prelabour CS, compared with vaginal birth, may be associated with additional increased risks. Prelabour CS exposes women to major surgery and the baby to the risks of birth before complete maturity, and it deprives both the benefits of spontaneous labour.

The question on the timing of the decision to conduct a CS (ie, whether it was made before or after the onset of labour pains) was first introduced in the Demographic and Health Surveys (DHS) in 2015. A previous single-country DHS analysis showed that prelabour CS, compared with vaginal birth, is associated with a higher risk of neonatal mortality (prevalence ratio $3.79,95 \%$ CI 1.03 to 13.93 , n=10 641 women).$^{12}$ However, to our knowledge, no multicountry analysis has examined prelabour CS using DHS data.

\section{OBJECTIVES}

Using nationally representative data from the DHS programme,${ }^{13}$ our objectives were:

- To estimate the proportion of prelabour CS among singleton, term deliveries without previous CS (considered lower risk women).

- To compare the rate of prelabour CS among lower risk women across countries by sociodemographic (eg, economic status, place of residence), childbirth (eg, place of delivery, size of baby) and antenatal care (ANC) (eg, number of visits, type of provider) characteristics.

- To assess association of prelabour CS, compared with vaginal birth, with perinatal outcomes (neonatal mortality, breastfeeding initiation, early skin-to-skin contact, duration of hospital stay).
- To compare the association of different types of caesarean birth (prelabour vs intrapartum CS) with perinatal outcomes.

\section{METHODS}

\section{Data source}

We searched the DHS programme website ${ }^{13}$ and retrieved data for LMICs with information on timing of CS, from DHSs conducted between 2015 and 2018. DHSs are largescale, nationally representative cross-sectional household surveys that are routinely conducted in LMICs to facilitate between-country comparisons. ${ }^{13}$ The surveys employ a two-stage, stratified cluster sampling design. ${ }^{14}$ In the first stage, each country is divided into geographic regions and the populations within the subnational regions stratified into urban and rural areas. These primary sampling units or clusters are selected with probability proportional to the contribution of that cluster's population to the total population. In the second stage, all households within a cluster are listed and a sample of households randomly selected for interviews by equal-probability systematic sampling. ${ }^{14}$ DHSs are considered the best available method of obtaining several types of health indicators in LMICs. ${ }^{15}$

\section{Study population}

We examined two study populations comprising women aged 15-49 years who reported having a childbirth in the last 5 years of the survey. The first population, named 'lower risk women' comprised singleton pregnancies at term in women with no previous CS. This group is similar to groups 1 to 4 of the Robson classification ${ }^{16}$. The second population comprised singleton pregnancies at term in women with previous CS.

\section{Exposure}

The DHS questionnaire asks women about pregnancy, antenatal and delivery care for live births in the past 5 years. The data on CS and other variables in the DHS are collected based on mothers' self-report. The selfreported data on timing of CS were collected by asking mothers, the question: 'When was the decision made to have the caesarean section? Was it before or after your labour pains started?' For this study, the exposure group comprised women who had a primary caesarean section (ie, first time CS) before the onset of labour pains and delivered a singleton infant at term. The unexposed group were women who had singleton, spontaneous, term, vaginal births in hospitals. History of a caesarean section before onset of labour pains was used as a proxy for prelabour CS.

\section{Outcomes}

The outcomes examined comprised early neonatal mortality (defined as reported death of a child within 7 days of delivery), neonatal mortality (death during the first 28 days of life), ${ }^{17}$ initiation of exclusive breastfeeding within 1 hour of birth, early skin-to-skin contact with baby (ie, skin-to-skin contact with mother immediately 
following birth for at least 1 hour ${ }^{18}$ ) and duration of hospital stay.

\section{Covariates}

We examined association of prelabour CS with a diverse set of maternal factors for the index pregnancy during the survey period: sociodemographic characteristics (maternal age at birth of child, parity, body mass index (BMI), economic status, education, place of residence, health insurance status); characteristics of childbirth (place of delivery, size of baby) and characteristics of ANC (number of visits, type of provider, time of first visit, location where ANC received). These factors were selected a priori based on the existing literature ${ }^{1920}$ and preanalytical assumptions of plausible associations with prelabour CS.

\section{Analysis}

Our statistical analysis is divided into three parts. First, we computed, for each study population (ie, singleton, term pregnancies with and without previous CS), average prelabour CS with 95\% CI for each country. We further disaggregated the average prelabour CS by asset-based household wealth quintiles (poorest, poorer, middle, richer, richest) and by place of residence (urban and rural). We then computed difference and ratio measures between richest and poorest quintiles, and urban and rural areas, to quantify absolute and relative differences in prelabour CS. Disaggregated proportions of wealth quintiles were presented using Box plots together with medians (mid-point estimates) and interquartile range.

Second, differences in the proportions of maternal factors (sociodemographic, childbirth, ANC characteristics) across delivery types (prelabour CS, intrapartum CS, vaginal delivery) were examined using $\chi^{2}$ tests. Association of delivery type with outcomes was estimated using odds ratios (ORs), calculated by multivariable logistic regression. ORs were adjusted for all sociodemographic, childbirth and ANC variables. Adjusted ORs (aORs) were estimated using variance-component (random intercept) multilevel logistic regression. The stratified multistage sampling design (women nested in clusters, nested in countries) and the survey normalised weights were taken into account in the analyses using the survey commands in Stata V.14.2 (StataCorp, College Station, Texas, USA).

Last, for each of the two study populations examined, we carried out sensitivity analysis using all pregnancies (ie, including preterm pregnancies). All statistical tests were two tailed and $p$ less than 0.05 was considered statistically significant. We used Stata V.14.2 (StataCorp) for all analyses.

\section{Missing data}

There were missing data on eight variables: size of baby at birth (6.7\% of the pooled sample), occupation (42.6\%), BMI $(30.0 \%)$, health insurance status $(10.8 \%)$, number of antenatal visits $(1.1 \%)$, ANC provider $(7.2 \%)$, first ANC visit (7.5\%) and ANC location (12.9\%). Missing values were excluded from logistic regression analyses. Further information on missing data for each variable is provided in the result tables.

\section{Patient and public involvement}

Patients and the public were not involved in the design and conduct of this research.

We followed the Strengthening the Reporting of Observational Studies in Epidemiology (STROBE) guideline ${ }^{21}$ in reporting this study.

\section{RESULTS}

We identified 26 LMICs that had DHSs with information on timing of CS (figure 1). Thirteen countries were from the African region (Angola, Benin, Burundi, Ethiopia, Guinea, Malawi, Mali, Nigeria, Senegal, Tanzania, Uganda, Zambia and Zimbabwe). Eleven countries were from the Asian region (Albania, Armenia, India, Indonesia, Jordan, Maldives, Nepal, Pakistan, Philippines, Tajikistan and Timor Leste). Two countries were from the American region (Colombia, Haiti).

Figure 2 shows the study profile. A total of 255227 women (singleton, term pregnancies, without previous CS) from

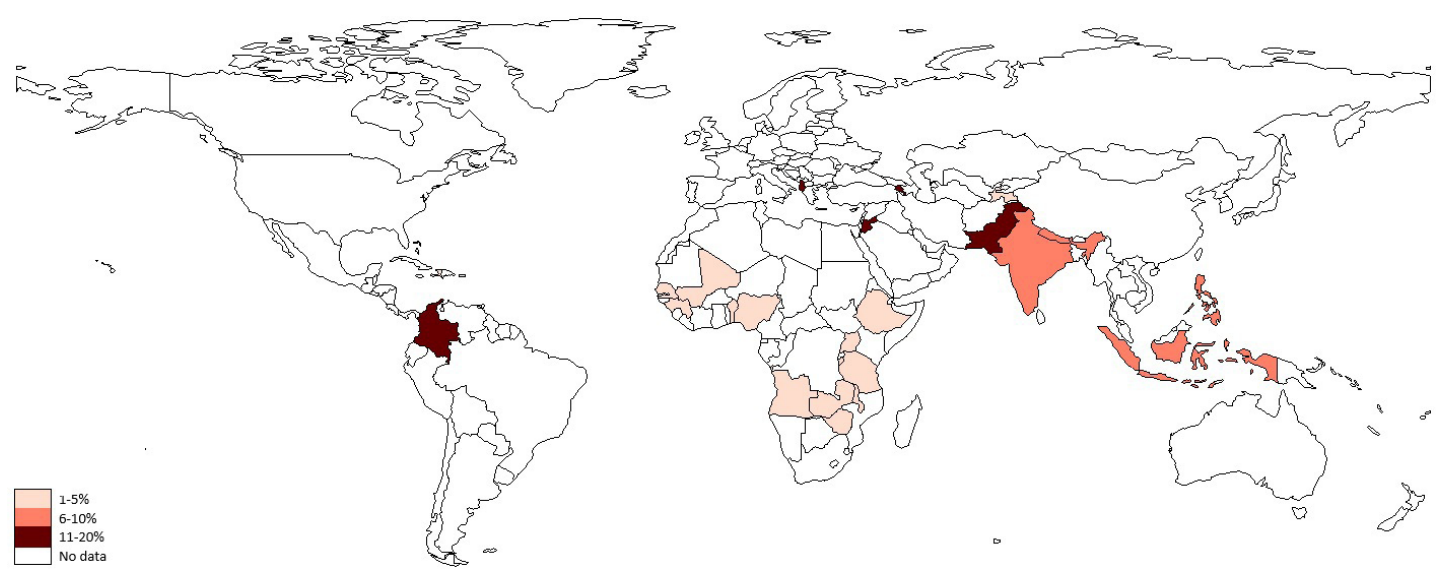

Figure 1 Twenty-six low-income and middle-income countries included in the analysis. 


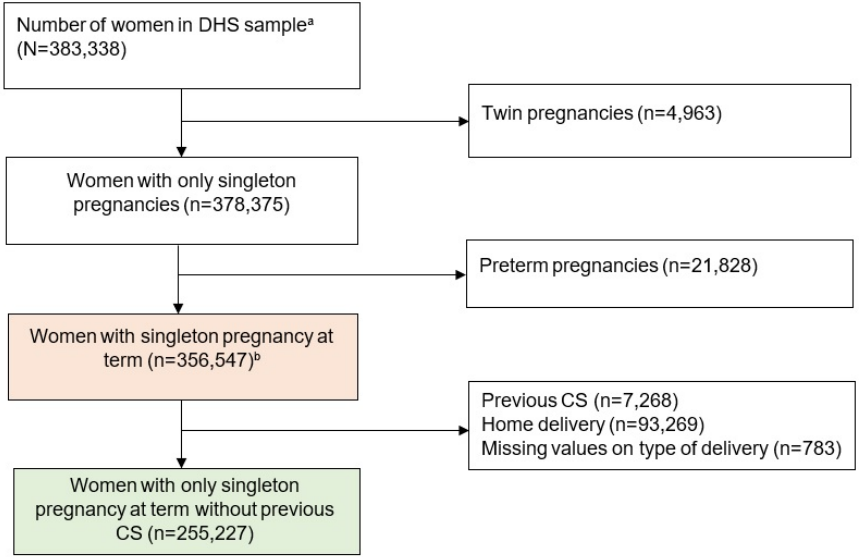

Figure 2 Flow diagram showing the selection of study

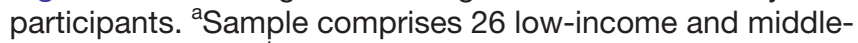
income countries; 'Including those with previous CS. CS, caesarean section; DHS, Demographic and Health Survey.

26 LMICs were included in the main analysis. The sample included 13 countries in Africa, 11 in Asia and 2 in America. The smallest sample ( $\mathrm{n}=1334$ women) was from Armenia and the largest $(\mathrm{n}=132103$ women) was from India (table 1). Average caesarean rates varied substantially, from $4.2 \%$ in Mali to $38.0 \%$ in Maldives. Average prelabour CS rates ranged from $1.3 \%$ in Zambia to $19.5 \%$ in Maldives.

Table 2 shows rates of primary prelabour CS by economic status across the 26 countries. Prelabour CS rates were lower in poorer women and tended to increase with rising economic status (figure 3). The median prelabour CS rate was $1.8 \%$ among women in the poorest quintile compared with $5.8 \%$ among women in the richest quintile. Substantial differences (exceeding 10 percentage points) in rates of prelabour CS between women in the richest and poorest quintiles were observed in seven countries (Albania, Indonesia, India, Colombia, Nepal, Pakistan and Philippines). Prelabour CS rate was higher among women in the poorest quintile compared with women in the richest quintile (4.6 percentage points difference) in one country (Maldives). In Africa, the largest difference was found in Burundi and Uganda (5.0 percentage points difference between the richest and poorest quintiles), while the largest ratio was in Nigeria, where prelabour CS was 37 times higher among women in the richest quintile compared with women in the poorest quintile $(3.7 \%$ in the richest quintile vs $0.1 \%$ in the poorest quintile). In Asia, the largest difference was found in Pakistan (18.9 percentage points between the richest and poorest quintiles) while Nepal showed the largest ratio, with prelabour CS in the richest quintile, 17.5 times higher than in the poorest quintile.

Table 3 shows rates of prelabour CS by place of residence across the 26 countries. Prelabour CS rates were higher in urban areas compared with rural areas in all countries, apart from Maldives, where rates were higher in rural areas (3.5 percentage point difference). The largest difference in urban-rural prelabour CS rates (exceeding
Table 1 Caesareans in women with singleton, term pregnancies without previous caesareans in 26 low-income and middle-income countries

\begin{tabular}{|c|c|c|c|}
\hline & $\begin{array}{l}\text { Total } \\
\text { number of } \\
\text { women }\end{array}$ & $\begin{array}{l}\text { Intrapartum } \\
\text { and prelabour } \\
\text { CS }\end{array}$ & $\begin{array}{l}\text { Average } \\
\text { primary } \\
\text { prelabour CS }\end{array}$ \\
\hline Country* & $\mathbf{N}$ & n (\%) & n (\%) \\
\hline \multicolumn{4}{|l|}{ Africa } \\
\hline Angola & 3879 & $235(6.1)$ & 75 (1.9) \\
\hline Benin & 6506 & 351 (5.4) & $118(1.8)$ \\
\hline Burundi & 6783 & $410(6.0)$ & 155 (2.3) \\
\hline Ethiopia & 2548 & $199(7.8)$ & $80(3.1)$ \\
\hline Guinea & 2737 & $148(5.4)$ & $72(2.6)$ \\
\hline Malawi & 10897 & 712 (6.5) & 145 (1.3) \\
\hline Nigeria & 8417 & 494 (5.9) & $204(2.4)$ \\
\hline Senegal & 6207 & $336(5.4)$ & $138(2.2)$ \\
\hline Mali & 4076 & $171(4.2)$ & $64(1.6)$ \\
\hline Tanzania & 4472 & $382(8.5)$ & $88(2.0)$ \\
\hline Uganda & 6165 & 475 (7.7) & $142(2.3)$ \\
\hline Zambia & 5706 & $290(5.1)$ & $73(1.3)$ \\
\hline Zimbabwe & 3752 & $245(6.5)$ & 87 (2.3) \\
\hline \multicolumn{4}{|l|}{ Asia } \\
\hline Tajikistan & 3532 & $194(5.5)$ & $90(2.5)$ \\
\hline Indonesia & 11164 & 2342 (21.0) & $879(7.9)$ \\
\hline Philippines & 5910 & 808 (13.7) & 471 (8.0) \\
\hline Timor Leste & 2183 & $168(7.7)$ & $96(4.4)$ \\
\hline India & 132103 & 22738 (17.2) & 12167 (9.2) \\
\hline Maldives & 2116 & 805 (38.0) & 413 (19.5) \\
\hline Nepal & 2219 & 300 (13.5) & $158(7.1)$ \\
\hline Pakistan & 4910 & 1094 (22.3) & 724 (14.7) \\
\hline Armenia & 1334 & 208 (15.6) & 174 (13.0) \\
\hline Jordan & 6165 & 1247 (20.2) & $862(14.0)$ \\
\hline Albania & 2208 & $598(27.1)$ & 424 (19.2) \\
\hline \multicolumn{4}{|l|}{ Americas } \\
\hline Colombia & 7376 & 2710 (36.7) & 1209 (16.4) \\
\hline Haiti & 1862 & $243(13.0)$ & $94(5.0)$ \\
\hline \multicolumn{4}{|l|}{ All countries } \\
\hline Total & 255227 & 37903 (14.9) & $19202(7.5)$ \\
\hline
\end{tabular}

${ }^{*}$ Categorised according to United Nations geographical grouping. CS, caesarean section.

five percentage points) was observed in five countries (Albania, Burundi, Colombia, India and Pakistan).

Table 4 shows characteristics of women with primary prelabour CS compared with primary intrapartum CS and vaginal deliveries. Overall, across the 26 countries, prelabour CS was higher among women aged more than 35 years, with higher education, residing in urban areas, with higher economic status, having health insurance, nulliparous women, with higher BMI, giving birth to very large 
Table 2 Primary prelabour caesarean sections by economic status in 26 low-income and middle-income countries*

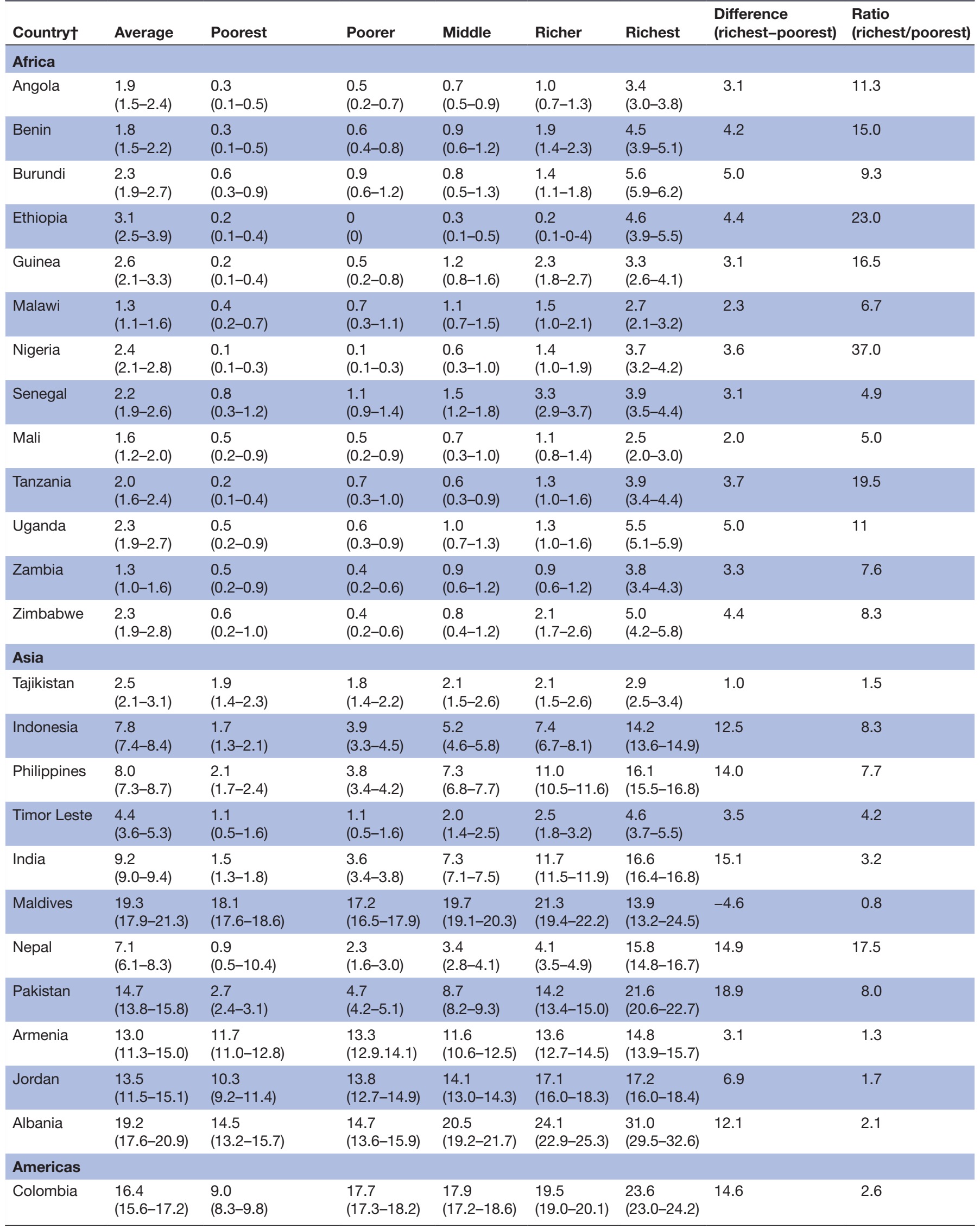


Table 2 Continued

\begin{tabular}{lllllllll}
\hline Countryt & Average & Poorest & Poorer & Middle & Richer & Richest & $\begin{array}{l}\text { Difference } \\
\text { (richest-poorest) }\end{array}$ & $\begin{array}{l}\text { Ratio } \\
\text { (richest/poorest) }\end{array}$ \\
\hline Haiti & 5.0 & 0.2 & 0.3 & 1.5 & 2.8 & 7.8 & 7.6 & 39.0 \\
& $(4.1-6.1)$ & $(0.1-0.5)$ & $(0.1-0.6)$ & $(1.2-1.9)$ & $(2.4-3.3)$ & $(6.9-8.7)$ & \\
All countries & & & & & & & & \\
Median $¥$ & - & 1.8 & 1.5 & 2.4 & 3.3 & 5.8 & - & - \\
& & $(0.7-5.6)$ & $(0.9-5.3)$ & $(1.1-8.8)$ & $(1.6-13.1)$ & $(4.1-16.8)$ & \\
\hline
\end{tabular}

*Values are percentages (95\% confidence intervals).

†Categorised according to United Nations geographical grouping.

$\ddagger$ Values in brackets indicate the interquartile range (middle $50 \%$ of estimates).

babies, in private facilities. Similar patterns of results were observed in stratified regional analyses, except for size of baby at birth (where higher rates were observed among women with very small babies in Africa) (online supplemental appendix 1: tables S1-S3).

Table 5 shows the characteristics of ANC in women with primary prelabour CS compared with primary intrapartum CS and vaginal deliveries. Overall, across the 26 countries, prelabour CS was higher among women who started ANC in the first trimester, had more than three $\mathrm{ANC}$ visits, where $\mathrm{ANC}$ was provided by a doctor, and in a private hospital. Similar patterns of results were observed in stratified regional analyses in Africa, Asia and America (online supplemental appendix 2: tables S4-S6).

Table 6 illustrates the results of adjusted multinomial logistic regression exploring association of maternal factors with primary prelabour CS. Factors significantly associated with increased odds of prelabour CS, compared with vaginal birth, were higher maternal age at birth, higher maternal education, urban residence, delivery in a private hospital, higher economic status, having health insurance, lower parity, higher BMI and larger size of baby. ANC factors significantly associated with increased odds of prelabour CS, compared with vaginal birth, were having more than three ANC visits, ANC by a doctor and receiving ANC from a private hospital. No

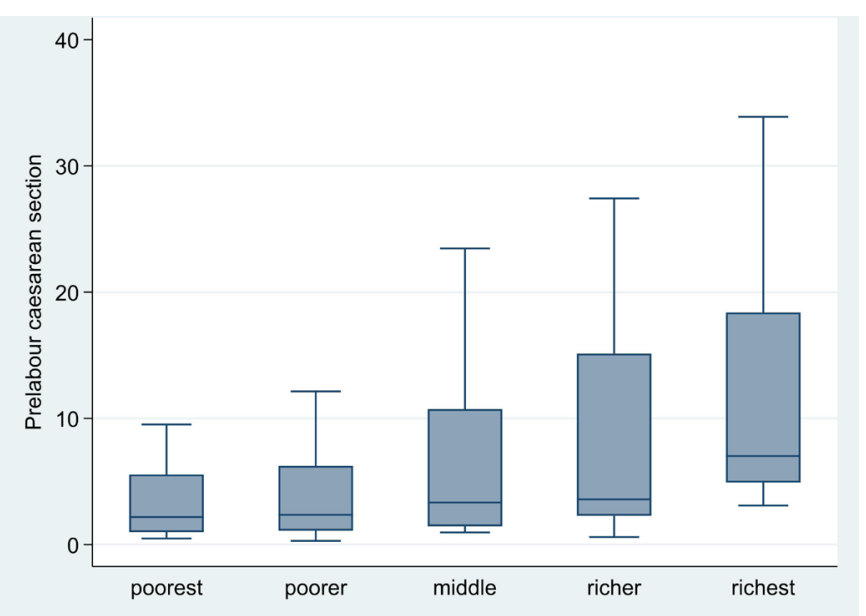

Figure 3 Primary prelabour CS rates by economic status in 26 low-income and middle-income countries. CS, caesarean section. significant association was found for timing of first ANC visit (table 7).

\section{Perinatal outcomes}

Table 8 shows association of primary prelabour CS with perinatal outcomes. Across the 26 countries, prelabour CS, compared with vaginal delivery, was associated with significantly increased odds of neonatal mortality, aOR $1.2,95 \%$ CI 1.0 to 1.5. However, no significant association was found for early neonatal mortality (aOR 1.2, 95\% CI 0.9 to 1.5 ). Compared with vaginal delivery, prelabour CS was associated with decreased odds of early skin-to-skin contact (aOR 0.4, 95\% CI 0.3 to 0.5 ), decreased odds of initiation of breastfeeding within 1 hour of birth (aOR $0.4,95 \%$ CI 0.3 to 0.5 ) and increased hospital stay more than 72 hours (aOR 6.6, $95 \%$ CI 5.9 to 7.4 ).

Region-specific associations of prelabour CS with perinatal outcomes are shown in online supplemental appendix 1: tables S7-S9. Across geographical regions, prelabour CS, compared with vaginal delivery, was associated with increased odds of neonatal mortality in Africa (aOR 2.2, 95\% CI 1.3 to 3.7). However, no significant association was found in Asia (aOR 1.2, 95\% CI 0.9 to 1.5 ) and Americas (aOR 2.4, 95\% CI 0.5 to 11.2). Similarly, prelabour CS compared with vaginal delivery, was associated with increased odds of early neonatal mortality in Africa (aOR 2.3, 95\% CI 1.3 to 4.1), but no significant association was found in Asia (aOR 1.2, 95\% CI 0.9 to 1.5 ) and America (aOR 2.4, 95\% CI 0.5 to 11.3). Compared with vaginal delivery, prelabour CS was associated with decreased odds of early skin-to-skin contact (aOR 0.2, $95 \%$ CI 0.1 to 0.3 ), decreased odds of initiation of breastfeeding within 1 hour of birth (aOR $0.2,0.1$ to 0.3 ) and increased hospital stay more than 72 hours (aOR 19.6, 95\% CI 14.1 to 27.4) in Africa. Similar trend of associations was found in Asia and Americas.

Similar patterns of results were found for analyses comparing association of different types of caesarean birth (prelabour and intrapartum CS) with perinatal outcomes, except for early neonatal mortality (where a significant association was found between intrapartum CS and early neonatal mortality in pooled data from 26 countries, aOR 1.7, 95\% CI 1.4 to 2.1) (table 8) and neonatal mortality (where a significant association was found between intrapartum CS and neonatal mortality 
Table 3 Primary prelabour caesarean section by place of residence in 26 low-income and middle-income countries* 
Table 3 Continued

\begin{tabular}{llcccccc}
\hline Country† & Average & Urban & & Rural & & $\begin{array}{l}\text { Difference } \\
\text { (urban-rural) }\end{array}$ & $\begin{array}{l}\text { Ratio } \\
\text { (urban/rural) }\end{array}$ \\
\hline Americas & & & & & & & \\
Colombia & $\begin{array}{l}16.4 \\
(15.6-17.2)\end{array}$ & 18.2 & $17.7-18.8$ & 11.6 & $10.6-11.7$ & 6.6 & 1.6 \\
Haiti & $\begin{array}{l}5.0 \\
(4.1-6.1)\end{array}$ & 7.2 & $6.6-7.9$ & 3.0 & $2.1-3.8$ & 4.2 & 2.4 \\
\hline
\end{tabular}

*Values are percentages (95\% confidence intervals).

†Categorised according to United Nations geographical grouping.

in the Asian region, aOR 1.7, 95\% CI 1.4 to 2.1) (online supplemental appendix 2: table S8).

\section{Ancillary analyses}

We conducted three sets of additional analyses. In the first set, we computed, for each country, average and disaggregated (by household wealth quintiles and place of residence) prelabour CS among women with previous CS (online supplemental appendix 2: tables S1-S3). Prelabour CS rates and differences between richest and poorest quintiles and urban and rural areas were generally larger, but followed similar patterns as those for women without previous CS.

In the second set of analysis, we explored associations of prelabour CS with various maternal characteristics restricted to women with previous CS (online supplemental appendix 2: tables S4-S11). We found similar pattern of results as those for women without previous CS, except for parity (where higher rates of prelabour CS was found among women of parity 1 to 2 with previous CS in Africa).

Findings for analyses comparing associations of prelabour CS with perinatal outcomes for women with previous CS were similar to those for women without previous CS, except for early neonatal mortality (where prelabour CS increased odds of early neonatal mortality among women with previous CS in pooled analyses across the 26 countries, aOR $1.6,95 \%$ CI 1.4 to 1.9 ) and neonatal mortality (where prelabour CS increased odds of neonatal mortality among women with previous CS in Asia, aOR $1.3,95 \%$ CI 1.1 to 1.6 ) (online supplemental appendix 2: tables S12-S15). Similar patterns of results were found for analyses comparing association of different types of caesarean birth (prelabour and intrapartum CS) with perinatal outcomes (online supplemental appendix 2: tables S12-S15).

In the third set of analysis of all pregnancies (term and preterm), prelabour CS was associated with increased neonatal mortality (aOR 1.2, 95\% CI 1.0 to 1.5 ) and early mortality (aOR 1.3 , 95\% CI 1.0 to 1.4 ) among women without previous CS (online supplemental appendix 1: table S10-S11). Similarly, prelabour CS was associated with increased neonatal mortality (aOR 1.3, 95\% CI 1.1 to 1.6) among women with previous CS (online supplemental appendix 2: tables S16 and S17). However, no significant association with early neonatal mortality was observed.

\section{DISCUSSION \\ Interpretation of results}

We examined nationally representative primary prelabour CS rates, risk factors and the relationship between prelabour CS with perinatal outcomes in 255227 lower risk pregnancies across 26 LMICs. Prelabour CS rates varied substantially across countries, ranging from $1.3 \%$ in Zambia to $19.5 \%$ in the Maldives. Differences across wealth subgroups were also significant, with the rate among women in the wealthiest subgroup about three times in the poorest subgroup. The highest prelabour CS rates and the most remarkable absolute differences between the richest and the poorest subgroups were observed in Asian countries. In contrast, countries from the African region had low prelabour CS rates and comparatively lower absolute differences between the richest and poorest subgroups. However, relatively large differences were also found in some African countries (eg, Angola, Benin, Ethiopia, Guinea, Nigeria, Tanzania, Uganda). The lack of a universal benchmark for optimal rate of prelabour CS, however, limits interpretation of our findings.

The low rates of prelabour CS in African countries and among women in poor and rural subgroups likely indicate inadequate access among women in need (underuse of CS). Conversely, higher rates of prelabour CS in some of the countries in Asia and the Americas and among women in wealthy and urban subgroups likely indicate overuse, possibly without medical indication.

Compared with vaginal births, babies born by CS prior to labour onset had a higher risk of dying during the neonatal period, reduced likelihood of early skin-toskin contact with mother, delayed initiation of exclusive breastfeeding and increased duration of hospital stay. Associations of prelabour CS with perinatal outcomes were largely comparable to those for intrapartum CS. This suggests that unnecessary caesareans are likely concealed both among prelabour and intrapartum caesareans (strategies, to reduce unnecessary caesareans, should, therefore, focus on preventing both avoidable prelabour 
Table 4 Characteristics of women with primary prelabour CS in the index pregnancy compared with primary intrapartum CS and vaginal deliveries in 26 low-income and middle-income countries

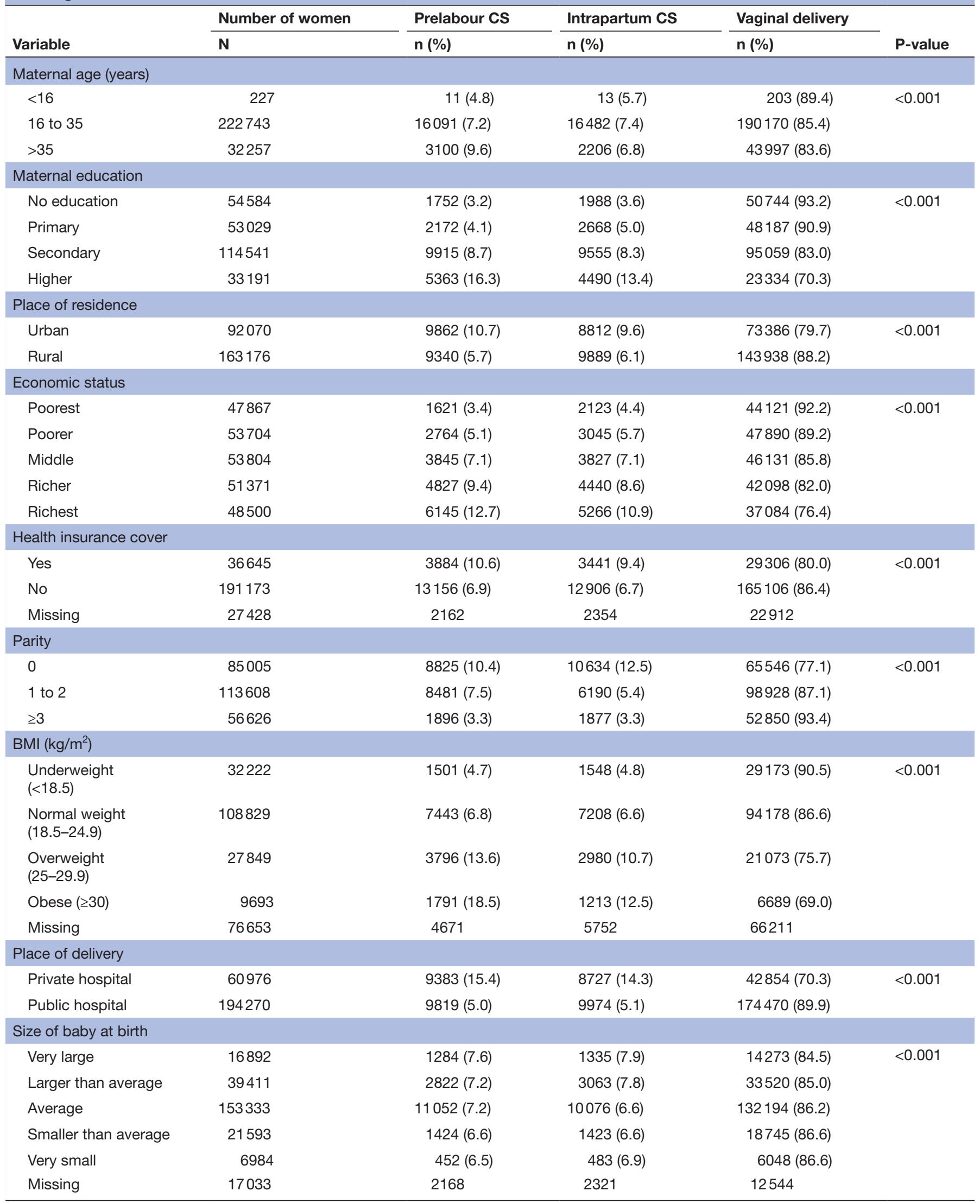

BMI, body mass index; CS, caesarean section. 
Table 5 Characteristics of antenatal care in pregnancies with primary prelabour CS compared with primary intrapartum CS and vaginal deliveries in 26 low-income and middle-income countries

\begin{tabular}{|c|c|c|c|c|c|}
\hline \multirow[b]{2}{*}{ Variable } & \multirow{2}{*}{$\begin{array}{l}\text { Number of women } \\
\mathbf{N}\end{array}$} & \multirow{2}{*}{$\begin{array}{l}\text { Prelabour CS } \\
\text { n (\%) }\end{array}$} & \multirow{2}{*}{$\begin{array}{l}\text { Intrapartum CS } \\
\text { n (\%) }\end{array}$} & \multirow{2}{*}{$\begin{array}{l}\text { Vaginal delivery } \\
\text { n (\%) }\end{array}$} & \multirow[b]{2}{*}{ P-value } \\
\hline & & & & & \\
\hline \multicolumn{6}{|l|}{ Time of first ANC visit } \\
\hline First trimester & 152798 & $14604(9.6)$ & $13380(8.8)$ & $124814(81.6)$ & $<0.001$ \\
\hline Second trimester & 72587 & $2997(4.1)$ & $3929(5.4)$ & $65661(90.5)$ & \\
\hline Third trimester & 10841 & $754(7.0)$ & $680(6.3)$ & $9407(86.7)$ & \\
\hline Missing & 19001 & 847 & 712 & 17442 & \\
\hline \multicolumn{6}{|c|}{ Number of antenatal visits } \\
\hline 0 & 18205 & $794(4.4)$ & $677(3.7)$ & $16734(91.9)$ & $<0.001$ \\
\hline 1 to 3 & 73915 & $3095(4.2)$ & $3734(5.0)$ & $67086(90.8)$ & \\
\hline$>3$ & 160260 & $15010(9.4)$ & $14092(8.8)$ & $131158(81.8)$ & \\
\hline Missing & 2847 & 303 & 198 & 2346 & \\
\hline \multicolumn{6}{|l|}{ ANC provider } \\
\hline Doctor & 107065 & 12888 (12.0) & 10794 (10.1) & 83383 (77.9) & $<0.001$ \\
\hline Nurse/midwife & 75516 & 3151 (4.2) & $4174(5.5)$ & 68191 (90.3) & \\
\hline Clinical officer & 27329 & 933 (3.4) & $1413(5.2)$ & 24983 (91.4) & \\
\hline Other (+TBA) & 26981 & 1419 (5.3) & $1629(6.0)$ & 23933 (88.7) & \\
\hline Missing & 18336 & 811 & 691 & 16834 & \\
\hline \multicolumn{6}{|l|}{ ANC location } \\
\hline Public hospital & 174868 & 10271 (5.9) & 10739 (6.1) & 153858 (88.0) & $<0.001$ \\
\hline Private hospital & 47498 & 7146 (15.0) & $6082(12.8)$ & 34270 (72.2) & \\
\hline Missing & 32861 & 1785 & 1880 & 29196 & \\
\hline
\end{tabular}

Clinical officer refers to non-physician clinician.

ANC, antenatal care; CS, caesarean section; TBA, traditional birth attendant.

and intrapartum caesareans). The results of the main analyses (lower risk pregnancies) were comparable to those of ancillary analyses restricted to higher risk pregnancies (ie, preterm and term pregnancies with previous CS), indicating that the observed associations are robust. The observed patterns of inequalities (across wealth, urban and rural subgroups), maternal risk factors and associations of prelabour CS with perinatal outcomes are consistent with those for overall CS reported in previous research. ${ }^{9} 1022-28$

A confluence of factors may contribute to the observed higher neonatal mortality with prelabour CS (compared with vaginal birth), including medical indications for the surgery (such as complications during pregnancy which may have been themselves the cause for conducting CS before labour), delay of a medically necessary CS or delayed breastfeeding initiation. ${ }^{29}$ However, in the absence of information on the medical indications for the CSs, we cannot infer causality between prelabour CS and the increase in neonatal mortality. A possible explanation for the delayed early skin-to-skin contact and initiation of breastfeeding is that caesarean delivery is usually accompanied by postoperative care routines, which might delay the baby's immediate contact with the mother. Separation of the baby from the mother immediately after birth may also be necessitated by limited space in the delivery room, particularly where delivery rooms are shared with other women, a common situation in many LMICs. Postsurgical pain experienced by mothers may result in discomfort, delaying initiation of breastfeeding. One previous cohort study found that women who had a caesarean delivery were three times more likely to have pain that affects breastfeeding than those who had a vaginal delivery. ${ }^{30}$

\section{Strengths and limitations}

To our knowledge, this is the first study to investigate the association between prelabour CS and perinatal outcomes in lower risk pregnancies, using the latest available, large, nationally representative data sets from LMICs. Furthermore, this is the first DHS analysis to distinguish between association of types of caesarean birth (prelabour vs intrapartum CS) with perinatal outcomes. Additional strengths include the use of multivariable analysis, simultaneously adjusting for multiple risk factors and confounders; and disaggregation of results by lower risk and higher risk groups, exploring potential interaction across obstetric subgroups.

Our results should be interpreted in the context of some limitations. First, our data set does not include all LMICs (eg, only two countries from the Americas, a region with high CS rates, ${ }^{10}$ were included in the analysis). Importantly, because of lack of recent national survey 
Table 6 Odds ratios calculated through multinomial logistic regression with vaginal delivery as the base outcome* $\dagger$

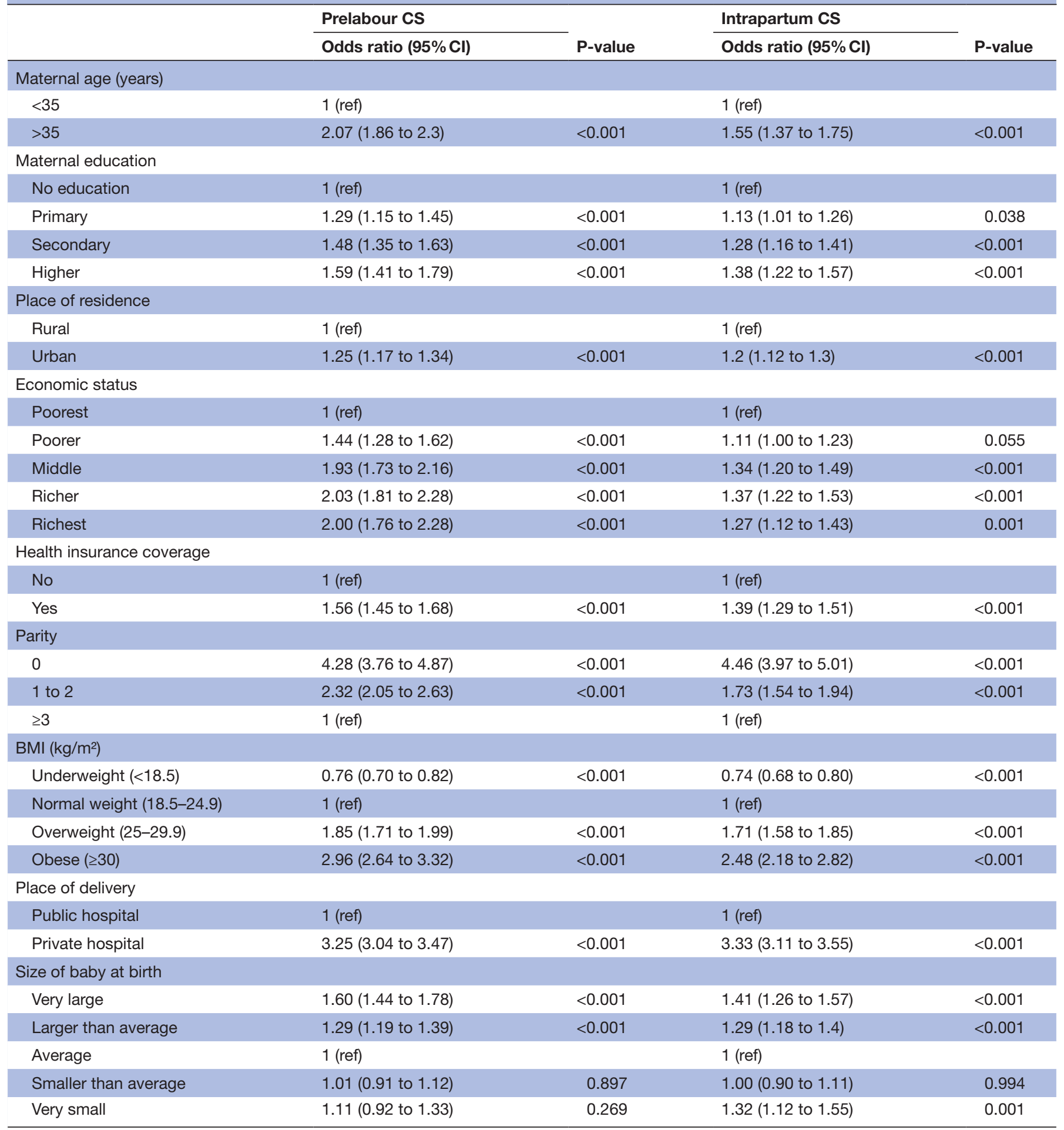

*Adjusted for country of survey, complex survey design (clustering, weights and stratification) and confounders (maternal age at birth, parity, education, antenatal care, place of residence, place of delivery, economic status, size of baby at birth).

†The results are based on 176069 participants with complete data for all covariates. 79158 (31\%) of 255, 227 participants had missing data and were excluded.

BMI, body mass index; CS, caesarean section.

data, the data set does not include some of the LMICs with the highest CS in the world, for example, Brazil with a national CS rate of $55.6 \%$ (in 2015) and China with a national CS rate of $41.3 \%$ (in 2016). ${ }^{10}$ This may limit the representativeness of our findings. Second, data from the DHS are self-reported based on a recall period of 5 years. We cannot, therefore, exclude the likelihood of measurement error for some of the factors analysed (eg, ANC 
Table 7 Odds ratios calculated through multinomial logistic regression with vaginal delivery as the base outcome* $†$

\begin{tabular}{|c|c|c|c|c|}
\hline & \multirow{2}{*}{$\begin{array}{l}\text { Prelabour CS } \\
\text { Odds ratio }(95 \% \mathrm{CI})\end{array}$} & \multirow[b]{2}{*}{ P-value } & \multirow{2}{*}{$\begin{array}{l}\text { Intrapartum CS } \\
\text { Odds ratio }(95 \% \mathrm{Cl})\end{array}$} & \multirow[b]{2}{*}{ P-value } \\
\hline & & & & \\
\hline \multicolumn{5}{|l|}{ Time of first ANC visit } \\
\hline Second trimester & 0.92 (0.86 to 0.99$)$ & 0.027 & 0.98 (0.92 to 1.05$)$ & 0.648 \\
\hline Third trimester & 1.09 (0.94 to 1.25$)$ & 0.244 & 0.92 (0.8 to 1.06$)$ & 0.228 \\
\hline 1 to 3 & 1 (ref) & & 1 (ref) & \\
\hline$>3$ & 1.57 (1.46 to 1.68$)$ & $<0.001$ & 1.41 (1.32 to 1.51$)$ & $<0.001$ \\
\hline \multicolumn{5}{|l|}{ ANC provider } \\
\hline Doctor & 1.61 (1.47 to 1.77$)$ & $<0.001$ & 1.42 (1.32 to 1.52$)$ & $<0.001$ \\
\hline \multicolumn{5}{|l|}{ ANC location } \\
\hline Public hospital & 1 (ref) & & 1 (ref) & \\
\hline Private hospital & 1.60 (1.51 to 1.69$)$ & $<0.001$ & 1.59 (1.50 to 1.69$)$ & $<0.001$ \\
\hline
\end{tabular}

Clinical officer refers to non-physician clinician.

${ }^{*}$ Adjusted for country of survey, complex survey design (clustering, weights and stratification) and confounders (maternal age at birth, parity, education, place of residence, economic status).

†The results are based on 212, 584 participants with complete data for all covariates. 42, 643 (16.7\%) of 255, 227 participants had missing data and were excluded.

ANC, antenatal care; CS, caesarean section; TBA, traditional birth attendant.

received, breastfeeding history). However, validation and reliability studies in several countries have shown that recall of CS is good in most settings. ${ }^{26} 3132$

Third, we could not adjust for all possible confounders due to a lack of detailed clinical information in the DHSs. For instance, the indication of prelabour CS, an important confounder, is not reported in the DHSs. Since prelabour CSs include both necessary and unnecessary caesareans, it is likely that a proportion of the 'lower risk women' needed a CS because of clinical or obstetric disorders (such as hypertension, pre-eclampsia or placenta previa) identified during pregnancy. In this subset of women, where prelabour CS was performed because of a medical complication, the adverse outcomes could be linked to the complication rather than the CS. We suspect this is particularly the case in the African region, where primary CS rates were generally low, suggesting that most women probably receive this procedure in response to obstetric complications, possibly the same underlying cause behind the observed increased odds of neonatal deaths. Since we could not assess the size of this subgroup of women with necessary prelabour CSs, caution should be exercised when interpreting the results to avoid the bias of reverse causality.

Fourth, the analysis relied on self-reported 'timing of decision to conduct caesarean section' as a proxy to the types of caesarean birth (prelabour and intrapartum CS). Although reasonable, the extent to which this proxy accurately measures actual types of caesarean births remain unclear. Fifth, large amount of missing data for some of the variables (eg, occupation (42\%), BMI $(30.0 \%)$ ) were excluded from the regression analyses. This reduced the precision of observed estimates. Finally, the populationlevel data analysed have the attendant limitations of observational studies, which preclude drawing any causal inferences for the relationship between prelabour CS and studied outcomes.

\section{Future research}

Prospective longitudinal studies are needed to understand better the determinants and the relationship between prelabour CS and perinatal outcomes, to provide a more solid basis for interventions aimed at optimising caesarean use. Future studies should collect and report data on indications of prelabour CS and explore the decision-making process for planned and emergency prelabour CS in LMICs. Further research into biologically plausible mechanisms by which prelabour CS may put an infant at higher risk for neonatal mortality are also needed. Directed acyclic graphs ${ }^{33}$ may aid these investigations. Long-term multisite studies of epigenetic consequences of prelabour CS and other modes of delivery are also warranted.

\section{Implications for policy and practice}

What can be done to mitigate the identified adverse effects of prelabour CS? Ideally, the adverse effects could be reduced 
Table 8 Association of primary prelabour CS with various outcomes in 26 low-income and middle-income countries

\begin{tabular}{|c|c|c|c|c|c|c|}
\hline Outcome & Total number (N) & n (\%) & OR $(95 \% \mathrm{Cl})^{*}$ & P-value & Adjusted OR $(95 \% \mathrm{Cl})$ & P-value \\
\hline \multicolumn{7}{|l|}{ Neonatal mortality } \\
\hline Vaginal delivery & 217324 & 2735 (1.3) & 1 & & 1 & \\
\hline Intrapartum CS & 18701 & $336(1.8)$ & $1.3(1.1$ to 1.5$)$ & $<0.001$ & 1.7 (1.4 to 2.0$)$ & $<0.001$ \\
\hline \multicolumn{7}{|c|}{ Early neonatal mortality } \\
\hline Intrapartum CS & 18701 & $299(1.6)$ & $1.4(1.2$ to 1.6$)$ & $<0.001$ & 1.7 (1.4 to 2.1 ) & $<0.001$ \\
\hline \multicolumn{7}{|c|}{ Early skin-to-skin contact } \\
\hline Vaginal delivery & 203648 & $112897(55.4)$ & 1 & & 1 & \\
\hline Prelabour CS & 16738 & $6097(36.4)$ & $0.5(0.4$ to 0.6$)$ & $<0.001$ & 0.4 (0.3 to 0.5$)$ & $<0.001$ \\
\hline Prelabour CS & 16738 & $8981(53.7)$ & $0.4(0.3$ to 0.5$)$ & $<0.001$ & 0.4 (0.3 to 0.5$)$ & $<0.001$ \\
\hline Intrapartum CS & 16098 & 7961 (49.4) & $0.3(0.2$ to 0.4$)$ & $<0.001$ & 0.4 (0.3 to 0.5$)$ & $<0.001$ \\
\hline \multicolumn{7}{|c|}{ Hospital stay $>72$ hours } \\
\hline Vaginal delivery & 212557 & $147749(69.5)$ & 1 & & 1 & \\
\hline Prelabour CS & 17992 & 17119 (95.2) & 8.6 (7.6 to 9.7$)$ & $<0.001$ & 6.6 (5.9 to 7.4 ) & $<0.001$ \\
\hline Intrapartum CS & 17200 & $16482(95.8)$ & 9.9 (8.8 to 11.1$)$ & $<0.001$ & 7.9 (7.0 to 8.9$)$ & $<0.001$ \\
\hline
\end{tabular}

${ }^{*}$ Adjusted for country of survey and complex survey design (clustering, weights and stratification).

†Adjusted for country of survey, complex survey design and confounders (maternal age at birth, parity, education, antenatal care, place of residence, place of delivery, economic status, size of baby at birth) [219566 observations].

$\mathrm{CS}$, caesarean section.

by promoting normal physiologic labour and birth in a safe and dignified environment for women with no indications for prelabour delivery. Proven evidence-based approaches to achieve this include prenatal birth preparation classes, ${ }^{34}$ psychoeducation for women with fear of pain, ${ }^{34}$ midwife-led continuity of care, ${ }^{35}$ encouraging free movement during labour $^{36}$ and continuous support during labour. ${ }^{37}$ Healthcare providers should be supported (eg, through in-service training and coaching) to develop and maintain skills to manage labour (including induction where appropriate and timely recognition and referral in remote facilities when prelabour CS is indicated) and to provide early breastfeeding and skin-to-skin support for women who have had CS. ${ }^{5} 18$ Along with improvement in quality of intrapartum care, financial incentives for use of prelabour CS should be removed.

Maternity care managers should explore new care routines to minimise the time of separation following caesarean birth. Father skin-to-skin care of the newborn is safe and effective ${ }^{38}$ and should be supported where separation of mother and newborn is medically indicated. Ensuring that the mother is as physically and emotionally comfortable as possible may help promote early initiation of breastfeeding. Facility coverage of early exclusive breastfeeding and skin-to-skin care of the newborn following caesarean birth should be routinely monitored and evaluated as quality of care indicators. These efforts should be accompanied with improvement in quality of care for small and sick newborns (who are the highest risk of mortality) and infrastructure and supplies to support early breastfeeding and skin-to-skin care (eg, adequate accommodation to allow rooming-in on a 24-hour basis). ${ }^{39}$

Evidence about risk factors and adverse outcomes associated with prelabour $\mathrm{CS}$ should help inform discussions about the choice of mode of delivery and may facilitate appropriate personalised birth planning and case management to mitigate the adverse effects of prelabour CS. The reasons for the observed variations in the frequency of prelabour CS across countries are context specific (underpinned by a complex interplay of factors at individual, societal and organisational levels). ${ }^{40}$ An understanding of country health systems, local norms and cultural practices and specific barriers and facilitators of concurrent overuse and underuse is crucial to developing country-appropriate strategies to optimise caesarean section use.

\section{CONCLUSIONS}

Primary caesarean section conducted before onset of labour, compared with vaginal birth, is associated with adverse perinatal outcomes in lower risk pregnancies in LMICs. Caesarean births should be monitored and audited regularly to determine appropriateness, provide opportunities for identifying context-specific factors and practices contributing to prelabour CS and promote optimal antenatal and perinatal care. 
Author affiliations

${ }^{1}$ UNDP-UNFPA-UNICEF-WHO-World Bank Special Programme of Research, Development and Research Training in Human Reproduction (HRP), Department of Sexual and Reproductive Health and Research, World Health Organization, Geneva, Switzerland

${ }^{2}$ Country Office, World Health Organization, Amman, Jordan

${ }^{3}$ Evidence-Based Healthcare Post-Graduate Program, Department of Medicine, São Paulo Federal University, Sao Paulo, Brazil

${ }^{4}$ Department of Social Medicine, Ribeirão Preto Medical School, University of São Paulo, Ribeirao Preto, São Paulo, Brazil

Acknowledgements We acknowledge the support of the Demographic and Health Survey Program (https://www.dhsprogram.com) for providing access to the data.

Contributors APB conceptualised the analysis with input from NO, SB, MRT and JPS. SB acquired the data and ran the analysis. NO prepared the first draft of the manuscript. NO, SB, MRT, JPS and APB participated in the interpretation of results, critical revision of the manuscript and approved the final version. NO is the guarantor.

Funding UNDP/UNFPA/UNICEF/WHO/World Bank Special Programme of Research, Development and Research Training in Human Reproduction (HRP), Department of Sexual and Reproductive Health and Research, World Health Organization, Geneva, Switzerland. The funders had no role in study design, data collection and analysis, decision to publish, or preparation of the manuscript.

Map disclaimer The inclusion of any map (including the depiction of any boundaries therein), or of any geographic or locational reference, does not imply the expression of any opinion whatsoever on the part of BMJ concerning the legal status of any country, territory, jurisdiction or area or of its authorities. Any such expression remains solely that of the relevant source and is not endorsed by BMJ. Maps are provided without any warranty of any kind, either express or implied.

Competing interests None declared.

Patient consent for publication Not applicable.

Ethics approval This study does not involve human participants; ethical approval was not required for this study. We used publicly available, non-identifiable secondary survey data. The DHS receives government permission and follows ethical practices including informed consent and assurance of confidentiality. Written consent to participate was obtained from respondents at the time of data collection by the DHS enumerators.

Provenance and peer review Not commissioned; externally peer reviewed.

Data availability statement Data are available in a public, open access repository. All data relevant to the study are included in the article or uploaded as supplementary information. Source data are available in a public, open access repository (https://dhsprogram.com/data/available-datasets.cfm). Data are accessible free of charge upon registration with the DHS Program. All data generated or analysed during this study are included in this manuscript and its additional files.

Supplemental material This content has been supplied by the author(s). It has not been vetted by BMJ Publishing Group Limited (BMJ) and may not have been peer-reviewed. Any opinions or recommendations discussed are solely those of the author(s) and are not endorsed by BMJ. BMJ disclaims all liability and responsibility arising from any reliance placed on the content. Where the content includes any translated material, BMJ does not warrant the accuracy and reliability of the translations (including but not limited to local regulations, clinical guidelines, terminology, drug names and drug dosages), and is not responsible for any error and/or omissions arising from translation and adaptation or otherwise.

Open access This is an open access article distributed in accordance with the Creative Commons Attribution Non Commercial (CC BY-NC 4.0) license, which permits others to distribute, remix, adapt, build upon this work non-commercially, and license their derivative works on different terms, provided the original work is properly cited, appropriate credit is given, any changes made indicated, and the use is non-commercial. See: http://creativecommons.org/licenses/by-nc/4.0/.

ORCID iDs

Newton Opiyo http://orcid.org/0000-0003-2709-3609

Maria Regina Torloni http://orcid.org/0000-0003-4944-0720
REFERENCES

1 Hanley GE, Munro S, Greyson D, et al. Diagnosing onset of labor: a systematic review of definitions in the research literature. $B M C$ Pregnancy Childbirth 2016;16:71.

2 Hundley V, Downe S, Buckley SJ. The initiation of labour at term gestation: physiology and practice implications. Best Pract Res Clin Obstet Gynaecol 2020;67:4-18.

3 Kenkel W. Birth signalling hormones and the developmental consequences of caesarean delivery. $J$ Neuroendocrinol 2021;33;:(:12912. Nov 3.

4 Buckley SJ. Executive summary of hormonal physiology of childbearing: evidence and implications for women, babies, and maternity care. J Perinat Educ 2015;24:145-53.

5 Who recommendations: intrapartum care for a positive childbirth experience. Geneva: World Health organization, 2018. Available: https://www.who.int/reproductivehealth/publications/intrapartumcare-guidelines/en/ [Accessed Apr 8 2021].

6 Paixao ES, Bottomley C, Pescarini JM, et al. Associations between cesarean delivery and child mortality: a national record linkage longitudinal study of 17.8 million births in Brazil. PLoS Med 2021:18:e1003791.

7 Dahlen HG, Downe S, Wright ML, et al. Childbirth and consequent atopic disease: emerging evidence on epigenetic effects based on the hygiene and EPIIC hypotheses. BMC Pregnancy Childbirth 2016;16:4.

8 Ortiz Jet al. Epigenetic and birth. Womens Health 2017;4:1-2.

9 Boatin AA, Schlotheuber A, Betran AP, et al. Within country inequalities in caesarean section rates: observational study of 72 low and middle income countries. BMJ 2018;360:k55.

10 Boerma T, Ronsmans C, Melesse DY, et al. Global epidemiology of use of and disparities in caesarean sections. Lancet 2018;392:1341-8.

11 Sobhy S, Arroyo-Manzano D, Murugesu N, et al. Maternal and perinatal mortality and complications associated with caesarean section in low-income and middle-income countries: a systematic review and meta-analysis. Lancet 2019;393:1973-82.

12 Yisma E, Mol BW, Lynch JW, et al. The changing temporal association between caesarean birth and neonatal death in Ethiopia: secondary analysis of nationally representative surveys. BMJ Open 2019;9:e027235.

13 DHS Program. Data from: the demographic and health survey program Repository. Available: https://dhsprogram.com/data/ available-datasets.cfm [Accessed 08 Apr 2021].

14 DHS. The demographic and health surveys (DHS) program, 2021. Available: http://dhsprogram.com/What-We-Do/Survey-Types/DHSMethodology.cfm [Accessed 6 Feb 2021].

15 Pullum TW. An assessment of the quality of data on health and nutrition in the DHS surveys, 1993-2003. methodological reports No. 6. Calverton, MD, USA: Macro International, 2008.

16 Robson classification: implementation manual. Geneva: World Health organization, 2017. Available: https://www.who.int/ reproductivehealth/publications/maternal_perinatal_health/robsonclassification/en/ [Accessed 30 December 2019].

17 World Health Organization. Neonatal and perinatal mortality: country, regional and global estimates. Geneva: World Health Organization, 2006.

18 Baby-Friendly Hospital initiative: revised, updated and expanded for integrated care. Geneva: World Health organization; 2009. 1.3, the global criteria for the BFHI. Available: https://www.ncbi.nlm.nih.gov/ books/NBK153487/

19 Khawaja M, Al-Nsour M. Trends in the prevalence and determinants of caesarean section delivery in Jordan: evidence from three demographic and health surveys, 1990--2002. World Health Popul 2007;9:17-28.

20 Khawaja M, Kabakian-Khasholian T, Jurdi R. Determinants of caesarean section in Egypt: evidence from the demographic and health survey. Health Policy 2004;69:273-81.

21 von Elm E, Altman DG, Egger M, et al. The strengthening the reporting of observational studies in epidemiology (STROBE) statement: guidelines for reporting observational studies. Lancet 2007;370:1453-7.

22 Harrison MS, Goldenberg RL. Cesarean section in sub-Saharan Africa. Matern Health Neonatol Perinatol 2016;2:6.

23 Lumbiganon P, Laopaiboon M, Gülmezoglu AM, et al. Method of delivery and pregnancy outcomes in Asia: the who global survey on maternal and perinatal health 2007-08. Lancet 2010;375:490-9.

24 MacDorman MF, Declercq E, Menacker F, et al. Infant and neonatal mortality for primary cesarean and vaginal births to women with "no indicated risk," United States, 1998-2001 birth cohorts. Birth 2006;33:175-82. 
25 Souza JP, Gülmezoglu A, Lumbiganon P, et al. Caesarean section without medical indications is associated with an increased risk of adverse short-term maternal outcomes: the 2004-2008 who global survey on maternal and perinatal health. BMC Med 2010;8:71.

26 Stanton CK, Dubourg D, De Brouwere V, et al. Reliability of data on caesarean sections in developing countries. Bull World Health Organ 2005;83:449-55.

27 Tefera M, Assefa N, Mengistie B, et al. Elective cesarean section on term pregnancies has a high risk for neonatal respiratory morbidity in developed countries: a systematic review and meta-analysis. Front Pediatr 2020;8:286.

28 Vogel JP, Betrán AP, Vindevoghel N, et al. Use of the Robson classification to assess caesarean section trends in 21 countries: a secondary analysis of two who multicountry surveys. Lancet Glob Health 2015;3:e260-70.

29 Edmond KM, Zandoh C, Quigley MA, et al. Delayed breastfeeding initiation increases risk of neonatal mortality. Pediatrics 2006;117:e380-6.

30 Albokhary AA, James JP. Does cesarean section have an impact on the successful initiation of breastfeeding in Saudi Arabia? Saudi Med J 2014;35:1400-3.

31 Tomeo CA, Rich-Edwards JW, Michels KB, et al. Reproducibility and validity of maternal recall of pregnancy-related events. Epidemiology 1999;10:774-6.

32 Tunçalp O, Stanton C, Castro A, et al. Measuring coverage in $\mathrm{MNCH}$ : validating women's self-report of emergency cesarean sections in Ghana and the Dominican Republic. PLoS One 2013;8:e60761.
33 Greenland S, Pearl J, Robins JM. Causal diagrams for epidemiologic research. Epidemiology 1999;10:37-48.

34 Who recommendations on non-clinical interventions to reduce unnecessary caesarean sections. Geneva: World Health organization 2018. Available: https://www.who.int/reproductivehealth/publications/ non-clinical-interventions-to-reduce-cs/en/ [Accessed Apr 8 2021].

35 Sandall J, Soltani H, Gates S, et al. Midwife-led continuity models versus other models of care for childbearing women. Cochrane Database Syst Rev 2016;4:CD004667.

36 Lawrence A, Lewis L, Hofmeyr GJ. Maternal positions and mobility during first stage labour. Cochrane Database of Systematic Reviews 2013:CD003934

37 Bohren MA, Hofmeyr GJ, Sakala C, et al. Continuous support for women during childbirth. Cochrane Database Syst Rev 2017;7:CD003766.

38 Ayala A, Christensson K, Christensson E, et al. Newborn infants who received skin-to-skin contact with fathers after caesarean sections showed stable physiological patterns. Acta Paediatr 2021;110:1461-7.

39 Setola N, Naldi E, Cocina GG, et al. The impact of the physical environment on intrapartum maternity care: identification of eight crucial building spaces. HERD 2019;12:67-98.

40 Betrán AP, Temmerman M, Kingdon C, et al. Interventions to reduce unnecessary caesarean sections in healthy women and babies. Lancet 2018;392:1358-68. 